\title{
Device to rehabilitate one's Physical and Learning Abilities
}

\author{
Jacek S. TUTAK, Wojciech KOŁODZIEJ
}

\begin{abstract}
This article presents an innovative device to rehabilitate people's physical and learning abilities. We aim to substantiate the subject, review existing solutions, and explain the most important issues connected with rehabilitation. We describe the CAD-based conceptual design of the device, its dedicated software, selection of actuators, and construction of the device's prototype, including its first tests. The device includes an original set of exercises, which can be individually set for every patient to improve his physical abilities, memory (both visual and auditory) and reflexes (i.e. reaction time to a stimulus). Rehabilitation exercises, which are performed in virtual reality and shown on a display screen, are additionally enhanced in real life by introducing obstacles and space restrictions, which the user faces while performing his tasks. Deliberate restrictions of one's space in which to perform exercises is made possible not only due to the device's original design, that is a four-frames construction, but also due to connectors with adjustable installation points, which enables positioning of obstacles to suit the current state of a patient. The prototype device and its original software have been designed to suit stroke convalescents and preschool children. First tests were carried out with preschool children and this article highlights the results of these tests. They included exercises, which were custom-made for children, such as a quiz, exercise to train one's reflexes, visual memory, auditory memory and games involving music. Since the rehabilitation device utilizes unique technical solutions, a patent application has been filed for it.
\end{abstract}

Keywords: hardware/software; neurological diseases; rehabilitation device; upper limb

\section{INTRODUCTION}

Stroke is the third most common cause of mortality across the world and is the leading cause of disability for people over 40 years old. Each year 2.5 million new cases of stroke are registered in Europe. Across the United States and Europe the annual incidence of stroke ranges between 110 and 290 cases per 100,000 people. In highly developed countries $31-50 \%$ of those who have suffered from a stroke do not regain independence, while $17-25 \%$ continuously rely on being taken care of by others. There exists a highly worrying statistical data which says that only about $12 \%$ of patients after stroke regain their full physical fitness in the affected limbs. It is expected that within the next $20-30$ years the number of people who suffer from stroke will double. Difficulties with regaining a full range of movement in the affected limb, and problems with concentration or with providing answers to simple questions are due to the loss of nerve connections in the damaged part of the brain, as a result of a neurological disease [1-3].

Those who have survived a stroke receive support through both traditional rehabilitation techniques and through rehabilitation carried out with the help of the latest mechatronic solutions [4]. One should start their rehabilitation after a stroke as soon as at all possible. The later the rehabilitation of one's cognitive and physical abilities start, the greater the risk of complications, which otherwise could have been avoided. The process and the final effect of one's recovery process largely depend on the pace of implementation of an individualized comprehensive rehabilitation program, which is adapted to the needs of a particular patient and relies on stimuli (biofeedback) in order to accelerate the process of rebuilding one's lost nerve connections [5-8].

For patients who can independently perform movements, and whose movements however are lacking in precision, a group of devices in support of rehabilitation performed in virtual reality has been devised, such as FysioGaming or Neuroforma. Through controlled movement of his body a patient can participate in a game displayed on a screen, using such aids as the above mentioned systems of motion detection and a range of cameras. The patient performs his tasks and/or rehabilitation exercises in the area close to his body and following instructions which occur only in virtual reality. While doing this he is not however able to factor in his movements any real obstacles or designated areas of space to which, for example, he would need to reach out. For persons who cannot carry out their exercises independently, additional solutions in support of movement have been designed. The Armeo system allows one to perform a series of non-weight bearing exercises to train their forearm and hand. Such exercises focus on grasping and squeezing, and enable the patient to move in 3D space while recording test results, modifying levels of difficulty and generating automated clinical reports. Some other examples of such solutions are the Amadeo or ReJoyce technologies [9-17].

The objective of this project was to develop a device which could diagnose and rehabilitate people who, as a result of a disease, have lost the full range of movements of their upper limbs and their movements' precision, who experience problems with concentration or with memory (visual and auditory), or with reflexes (time of producing a correct response to a stimulus), especially those affected with a slight hemiparesis [18-20].

\section{THE DESIGN AND THE OPERATIONAL PRINCIPLE OF THE DEVICE}

The device consists of a wooden base with attached four wooden frames, which are $25 \times 25 \mathrm{~cm}$ long. Onto the inner side, between the top and the bottom part and between the left and the right part of each frame six sets of sensors are placed, which detect the interruptions of the IR light beams (on one side there is a transmitter, and on the other is a receiver). The user performs the exercises by inserting his hand in a frame and thus interrupting a IR beam [21].

On top of each frame, there is a LED diode and a frame number. Whenever the IR sensor detects a hand within its operating field, the LED diode lights up thus providing a feedback for the user, telling him about the IR beam being 
interrupted. The LED diode not only informs the user about the interruption of the IR beam but it also introduces a biofeedback element to his rehabilitation (i.e. a visual stimuli).

A touch-screen has been mounted in the central part of the device, between the numbers of the two adjacent middle frames. The screen displays the commands for ongoing exercises and displays rehabilitation reports. More detailed information about the software is provided further down in this article [22-24].

A set of vision cameras has been mounted above the touch-screen. The cameras enhance the rehabilitation through additional features, such as an automatic detection of the user, logging the user in without the need to manually select him from a pre-determined list, and enabling to control the positioning of one's upper limbs while interrupting the IR beam between the frames.

As an option, a keyboard with a mouse and speaker can be attached to the base of the device. The keyboard and mouse are an alternative to a touch screen display, and are used for example to support the patient database. The sounds from the loudspeaker are used in selected exercises as a feedback for the patient, e.g. regarding the correct manner of breaking the IR beam or for the games with the use of music. Sounds are just another way of providing stimulation, which is so important for biofeedback.

Below the touch screen, half the height of the connector between the two adjacent middle frames, there is a proximity sensor, which measures the distance between the device and the user. The distance between the user and the device is very important in rehabilitation since a user who sits closer to the device engages different muscles (not necessarily these that at this point have to be diligently rehabilitated) than if he sits at a bigger distance. The device also indicates this feature. The distance sensor together with a dedicated software solution is essential in the proper diagnosis of the user's current state of health and to report the progress of his rehabilitation.

In order to enhance the process of rehabilitation, an option for individualized restriction of one's space to perform the exercise has been introduced. Two coaxial couplings mounted to the inner sides of each frame enable inserting removable connectors.

The conceptual design of the device has been developed with the use of the CAD computer technology. Autodesk Inventor 2017 and built-in 3D design tools were used to develop the project. This whole device to rehabilitate one's physical and learning abilities is shown in Fig. 1.

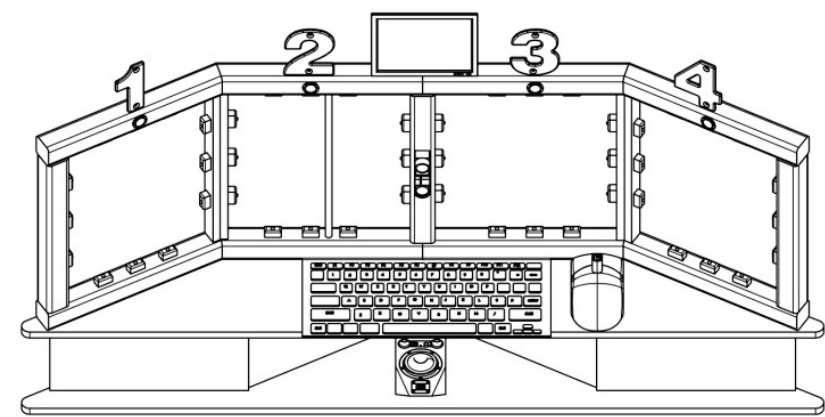

Figure $1 \mathrm{~A}$ sketch of the CAD-based device to rehabilitate one's physical and learning abilities
The device's operation is controlled by a central unit, which uses the Raspberry Pi model B microcomputer, and specialized original software, which enables patient's recovery and exercising. The control system and the power supply are located in a drawer at the bottom of the structure.

\section{KEY ELECTRICAL COMPONENTS AND HOW TO ASSEMBLE THEM}

The design uses LED diodes, $10 \mathrm{~mm}$ in diameter, in four colours (red, yellow, blue and green), where each colour is assigned to a different window in the device. The sensor, which the device is equipped with, is a IR diode of $3 \mathrm{~mm}$ with the illumination angle of 10 degrees. The sensors can be powered with $3.3 \mathrm{~V}$ or $5 \mathrm{~V}$ voltage (the $5 \mathrm{~V}$ option will have a bigger scope). The receivers have a transistor output of the open collector type.

Also, the device uses a 7" touch screen with $800 \times 480$ px resolution, which is a dedicated version meant for the Raspberry Pi microcomputers. The set includes a controller responsible for the touch recognition and for video transferring. The screen uses a DSI connector, which connects the screen controller with the ribbon cable, which is supplied. Raspberry Pi model B microcomputer controls the electronic components due to its microcontroller capabilities. A GPIO connector, which is mounted on the board, can be used for sensors connecting. The Raspberry provides an ideal solution for this project by enabling working with LINUX, programming in C or in Phyton. All electrical components in the first model have been connected to the contact plate, which enables the creation and testing of electronic systems without having to solder. A power supply module to the contact plate XD-42 is used to power electronics, which allows connecting to $5 \mathrm{~V}$ or 3.3 $\mathrm{V}$ power supply. This module can be powered from a mini USB connector with $5 \mathrm{~V}$ voltage or from a AC adapter with $12 \mathrm{~V}$ voltage. After assembling sensors and electronics, the power supply had to be connected from the left drawer to the right one, where electronics is placed. Fig. 2 shows how all the components got connected.

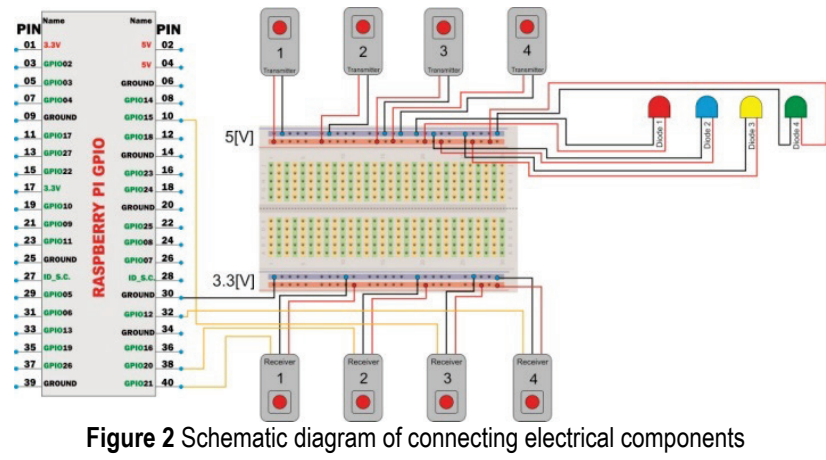

\section{SOFTWARE}

The original application was written in Python under the Linux platform for the Raspberry Pi microcomputer. Python is a high-level language for general purposes, it includes a comprehensive suite of libraries, and therefore can be used both for education and in commercial developments. It is characterized by its brevity and readability of the source code. Python is an interpreted 
language, i.e. a programmer can write a script or a program and execute it immediately with the compilation into machine code [25-27].

A set of pyQT libraries was used to build the device's graphical interface, enabling the user to create friendly graphical interfaces for programs written in Python. QT Creator was used to facilitate positioning of elements in the graphical interface. It contains predefined functions, classes, and objects, such as buttons, forms, text boxes, etc. Fig. 3 shows the user interface for the main menu after pressing the "Games for Kids" and "Rehabilitation after a stroke" button, from which the user selects his exercises [28].

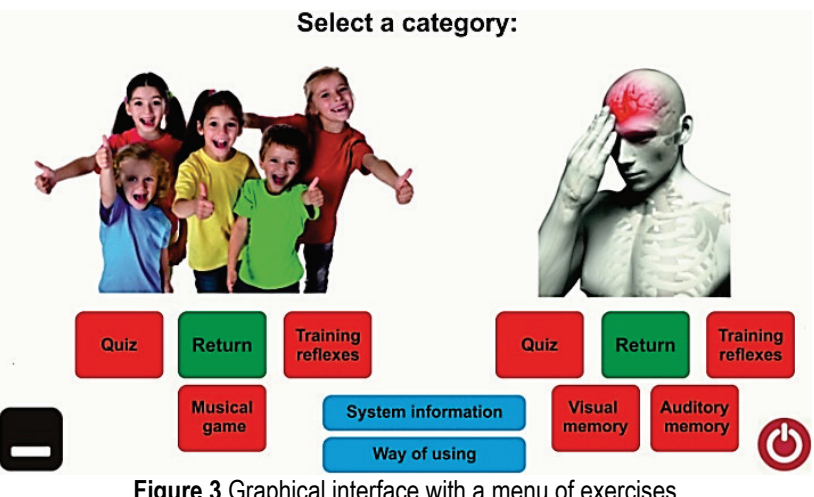

Figure 3 Graphical interface with a menu of exercises

The set of games for children includes:

- Quiz,

- Exercises to train one's reflexes,

- Games with the use of music.

The set of games for people after strokes includes:

- Quiz,

- Exercise to train one's reflexes,

- Visual memory,

- Auditory memory.

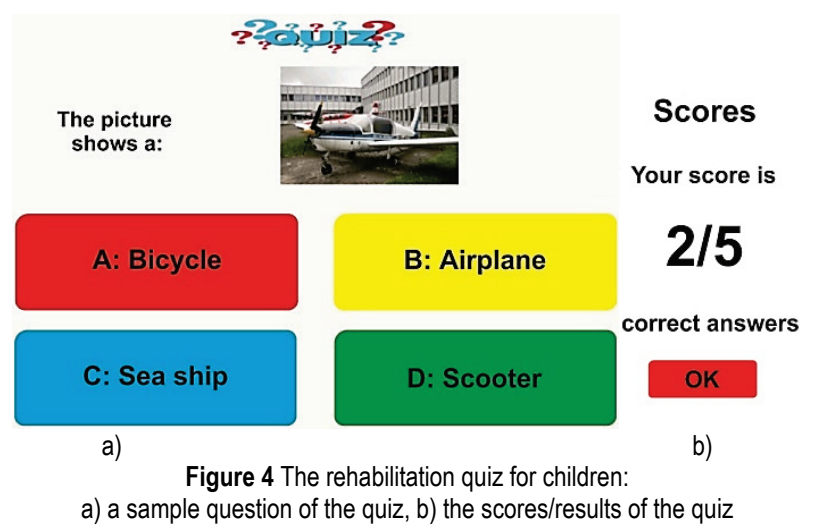

A quiz was one of the first games, which were prepared for both the stroke convalescents and children. Its content varies depending on the target group, but the very idea and the way to play the game remain the same. The quiz contains a set of questions which have been developed especially for the target group (including for persons who have been neurologically impaired) accompanied with four answers to choose from, one of which is the correct one and which can be selected by inserting one's hand into the corresponding window frame and thus breaking the IR beam. Every answer displayed on the screen appears against a different colour background, the colour matching that of the LED diode mounted on the frame. In this particular exercise, special focus was put on using the visual biofeedback.

Another exercise is to train the reflexes of the user that is the time between a stimulus and a response. The user's job is to follow commands, which tell him to break the IR beams in frames with particular numbers. A computer program randomly generates numbers from 1 to 4 , where each number indicates a frame with a sensor. The user has to break the beam in the shortest possible time. After the exercises (of 10 randomly chosen numbers), an average response time is shown, in milliseconds (Fig. 5 and Fig. 6).

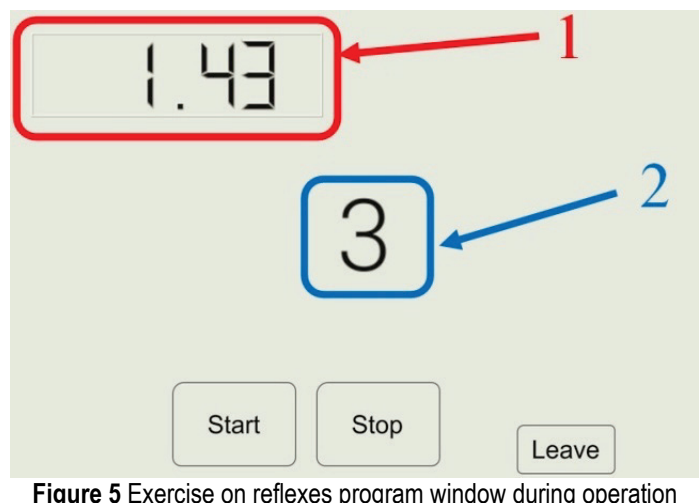

Where: 1 - is a window which shows time from generating a random number to breaking the correct IR beam; 2 - is a window in which a random number between 1 and 4 is displayed; 3 - is the result in milliseconds.

It should be highlighted that it is possible to set the time to display the number, which the user is expected to react to. For example, children were given the period of 2 seconds, while for the stroke patients the time was extended to 20 seconds due to the existing limitations of their movements.

$$
\text { เ. แ4 }
$$

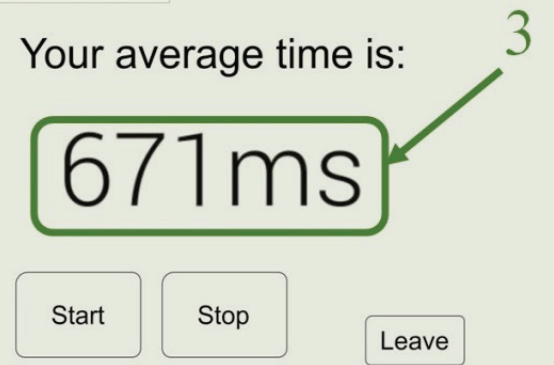

Figure 6 Exercise on reflexes after generating final results

The last exercise in the children's tab is a game with music. This game consists in making music with one of the selected instruments (including a guitar, piano, or drums) by breaking the IR beams between the frames. One can also choose some background music. This exercise aids the recovery process by composing music. Fig. 7 shows the application's main window. The Raspberry Pi's pins have been set as input and are connected to an internal resistor. The pins respond to a falling edge and are connected to sensors, which detect when an IR beam is broken. Similar definitions exist for classes, which use sensors detecting 
the moment when an IR beam is broken. Audio playing features are based on the type of instrument and the number of the bundle, which gets broken. Sounds are stored in files with the .wav extension on the Raspberry Pi microcomputer's memory card.

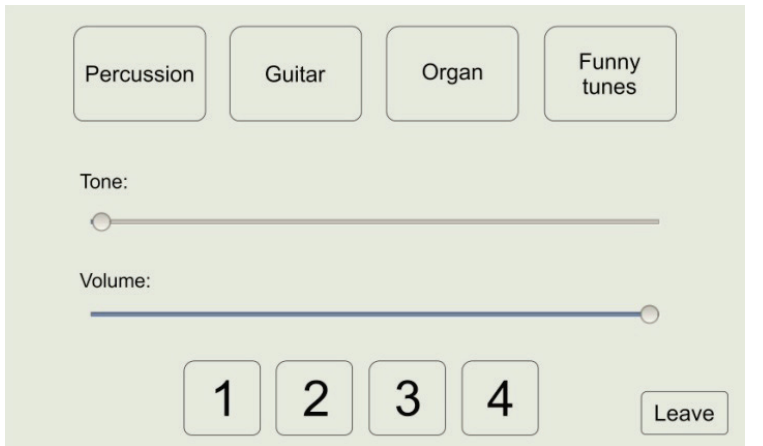

Figure 7 Application window for the "Musical Game"

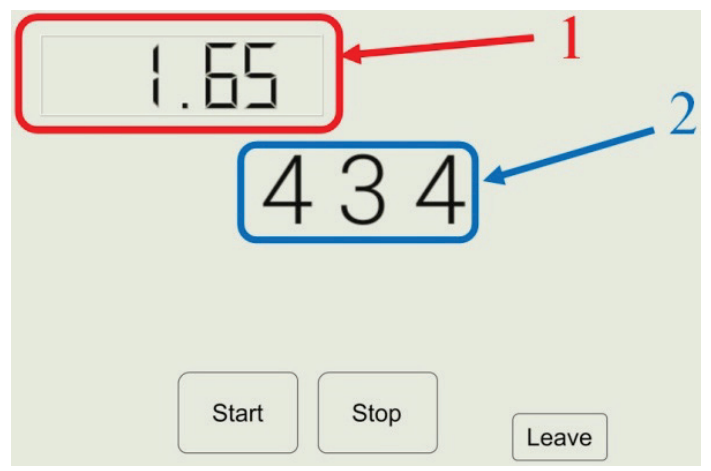

Figure 8 Memory exercise: program window during operation

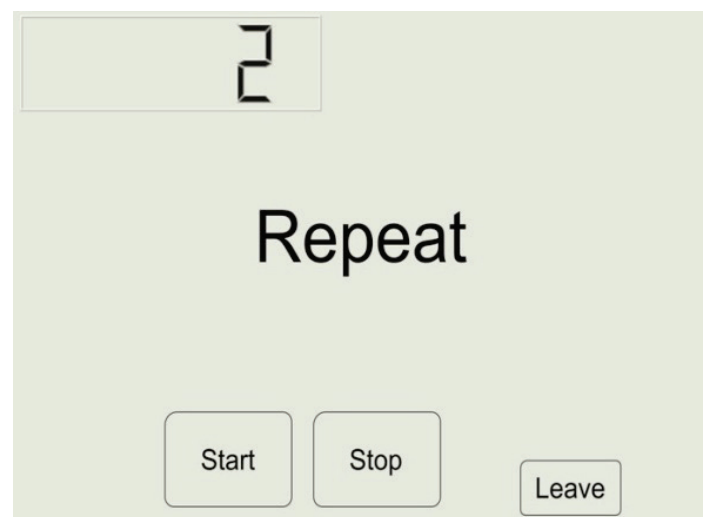

Figure 9 Memory exercise program window after exceeding the time of $2 \mathrm{~s}$ which was the time limit to produce an answer

Where: 1 - is a window which counts down the time limit of 2 seconds; 2 - is a window with the generated sequence of three numbers (Fig. 8).

One exercise which has been designed only for stroke convalescents is the rehabilitation of the user's memory. This exercise consists in memorizing a series of randomly generated numbers between 1 and 4, which are displayed on the screen, where each number corresponds to a number of a frame with a sensor. The user is then given two seconds to memorize the sequence of the generated numbers. Then the series of numbers is concealed and the user's task is to re-create the series by breaking the adequate IR beams.

Similarly, the sound memory training follows the pattern of the visual memory training. This exercise is based and puts a particular focus on the audio biofeedback.
The principle, which governs this program, is similar to the program, which trains one's memory using number sequences. In this case, the user's task is to memorize and then to replay the proper sequence of sounds which have been assigned to frames. Before starting the exercise one can see which sound corresponds to which frame. On pressing the "Start" button, (Fig. 10 and Fig. 11) three notes are played. The user's task is to play them in the same sequence.

\section{Press Start}

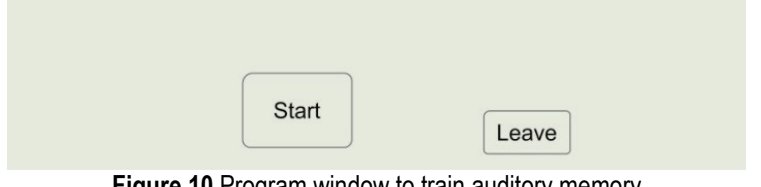

Figure 10 Program window to train auditory memory

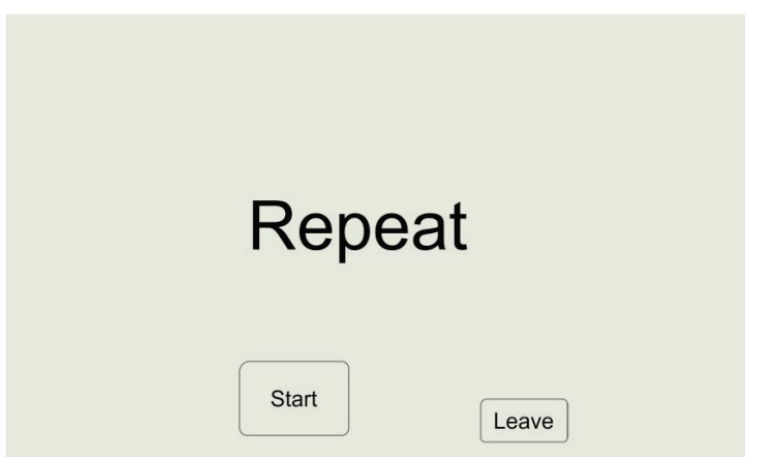

Figure 11 Program window to train auditory memory

The program randomly generates a number between 1 and 4. Each of the four numbers is assigned to a sensor and to a corresponding sound.

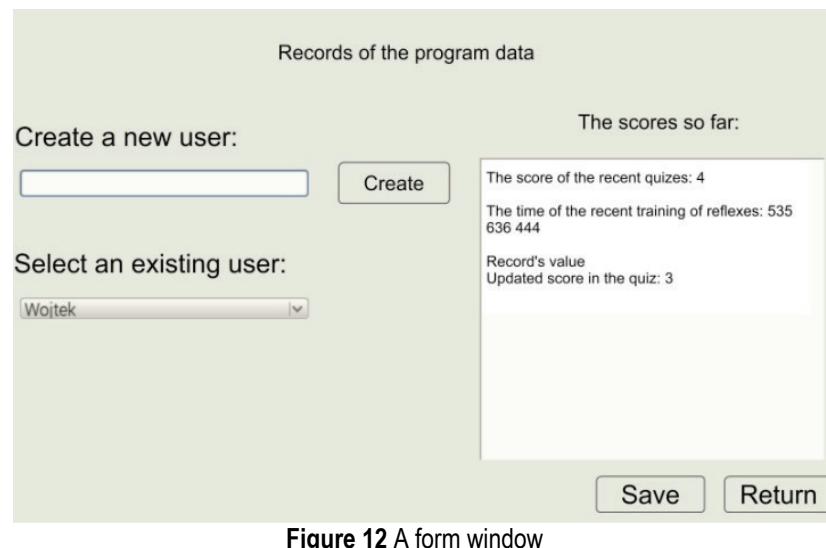

Unlike in games and activities for children, scores in the rehabilitation programs are both displayed and saved to a text file, using a special form. This solution facilitates monitoring of the patient's progress. The form allows adding a new user, selecting an existing user, reading previous results, and saving the results to a text file on microcomputer's memory card. The form is displayed 
every time an exercise is completed. Fig. 12 presents a form window.

The program opens the text file and feeds the user data, which are stored as a list in the program's variable. This list enables distinguishing between individual users in the file. Another function displays the results, which were obtained during previous exercises and in the current exercise. The function receives the result and the information, which specifies what it refers to, and whether it is the result of a quiz or of an exercise to train one's reflexes. Another feature of the program enables adding a new user to the database. By pressing "Save" the user creates a new position in the text file. One last important element is the function, which enables saving current results to a file. By pressing "Save" a new value is added to the list of the quiz results or the exercise on reflexes of a given user.

After selecting the "Way of using" button from the main menu, a window appears which includes information about the station's use. The screen shows a button to return to the previous screen, as well as the user instruction, which is stored as a picture with the tiff extension. Fig. 13 shows a screen displaying information on the way of using the station.

\section{IMPORTANT!}

\section{The pictures below show the correct position} of your hand in a frame with a sensor

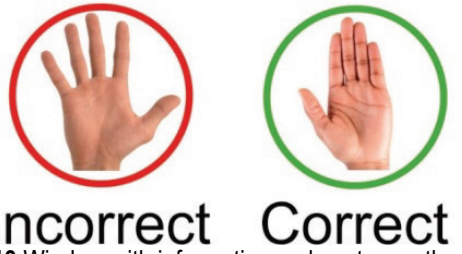

Figure 13 Window with information on how to use the station

After pressing the "System Information" button from the main menu, a screen is displayed which contains a text box with a button to return to the previous screen. The text is formatted as HTML and contains information about the system, its version, and information about the author of the program.

The system is very versatile and it should be highlighted that each of the presented exercises can easily be adapted to a different group of patients, with differing base reasons for dysfunctions of their upper limbs. The universal nature of the system also shows through the fact that even its first prototype includes an additional children's tab for educational program, which is aimed at preschool children. Also, every exercise which the system contains can be freely edited and developed by the teacher, thus enabling knowledge in an interesting and unconventional way, with the use of physical exercises. Each exercise can be repeated many times over. It is possible to individually set levels of difficulty and to bring up the reports from any given period.

\section{THE MAKING OF THE PROTOTYPE}

The construction is made with high quality wood and aluminum tubes, $8 \mathrm{~mm}$ in diameter. The whole construction weights approximately 7 kilos. After putting all the elements together, the whole structure was varnished. The next step was to mount the LEDs, then the IR beam sensors, which were fastened with screws of $2 \mathrm{~mm}$ in diameters. Tests proved that the sun light negatively affected the sensors' performance. Therefore, relatively small lenses were chosen in order to limit solar penetration into them, a decision that significantly improved the sensors' performance. The Raspberry Pi microcomputer was mounted in the right drawer. Then the Raspberrydedicated display screen was mounted. Finally, cables were fixed. They lead to the rear of the structure and have been placed in a space especially provided for electronics. Fig. 14 shows the final result of the work on the station.

The prototype's final budget proves that the initial plans to keep the costs down were successful. The price of components used for the prototype is 250 euro. This costing does not include the time spent on construction or the cost of creating the software. It is worth mentioning that prices of medical devices which are currently on the market are between dozen thousand to a few tens of thousands euro.

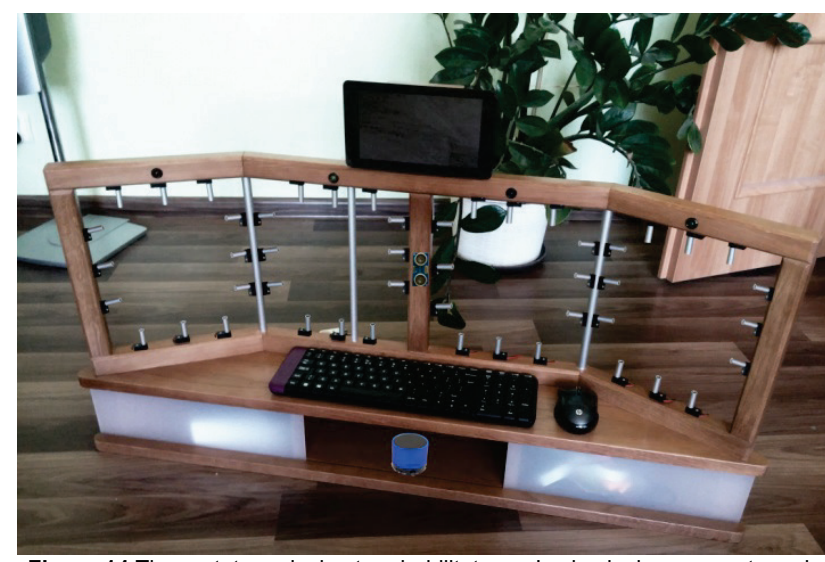

Figure 14 The prototype device to rehabilitate one's physical movements and learning abilities

\section{TESTING OF THE DEVICE}

Because of its versatile nature, the first tests of the device were carried out in a group of preschool children. The aim of the tests was, among other, to check whether the device meets the design's objectives, which is:

- the station's mobility,

- $\quad$ its versatile character, i.e. the ability to easily change over to adjust to the current demand, for example to rehabilitate patients with other diseases, to create a stand to educate children, etc.

- an intuitive user interface which stores information about the progress of the therapy, as it increases one's self-confidence and interest in the exercise.

The children were mostly interested in the exercise to train the response time and in the educational quiz. Those who participated in testing of the device said that it was a good alternative to a standard computer, since it positively affected children's development through games. The verification and testing of the device were carried out in a kindergarten, with children aged between 5 - 7 years of age. Both children and teachers took a great interest in the device. Also, the control system with the use of sensors and 
breaking the IR beams seemed very attractive and mobilized children to want to participate in exercises. Fig. 15 and Fig. 16 show a photo from testing.

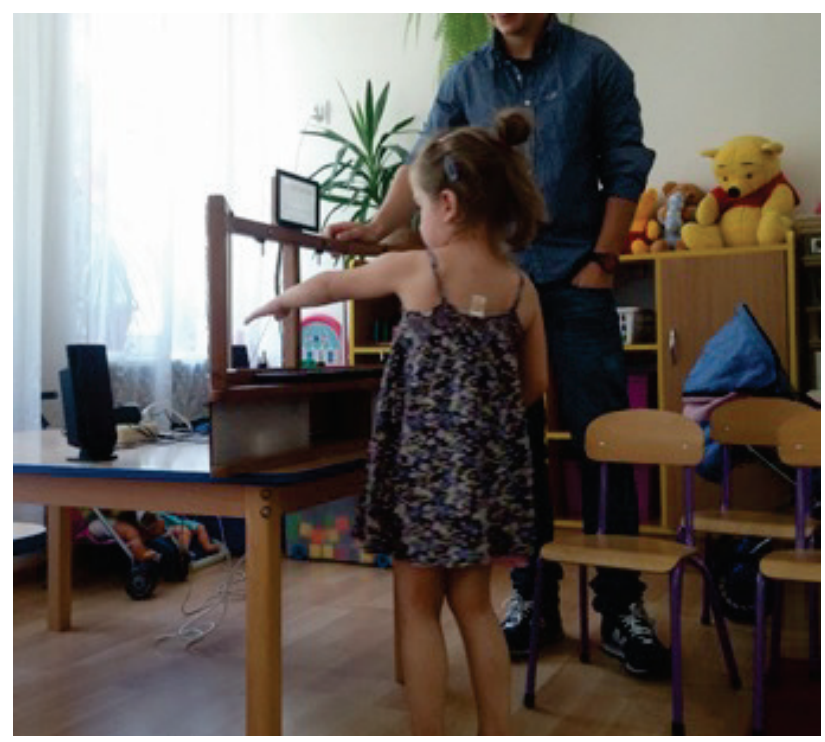

Figure $15 \mathrm{~A}$ preschool age child tests the device

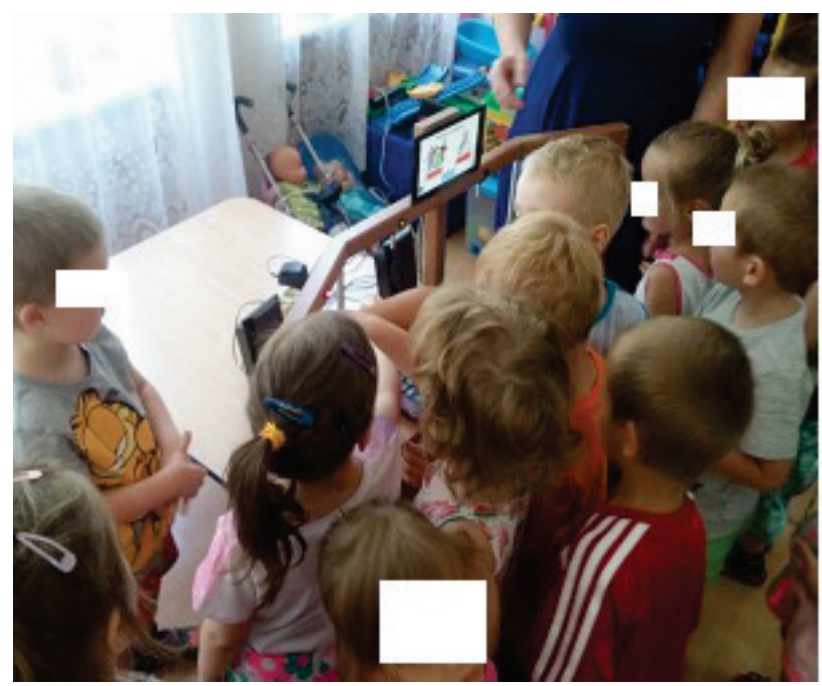

Figure 16 Several youngest are testing the device

The figures below show the results of the first tests.

Quiz

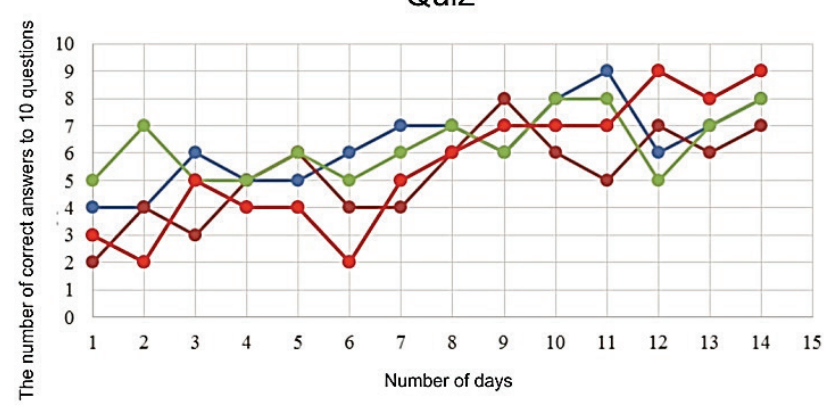

$\longrightarrow$ Person $1 \longrightarrow$-Person $2 \longrightarrow$ - Person $3 \longrightarrow$ Person 4

Figure 17 Results of memory training exercises

Also, rehabilitation exercises were verified in a group of four persons. The study consisted in performing selected exercises once a day for a period of 14 days. The first graph (Fig. 17) shows how the number of correct answers changed over time, and that for every participant the trend was growing.

The second graph (Fig. 18) displays the average response time in milliseconds and shows that towards the final stage all the people achieved a similar result of 600 ms.

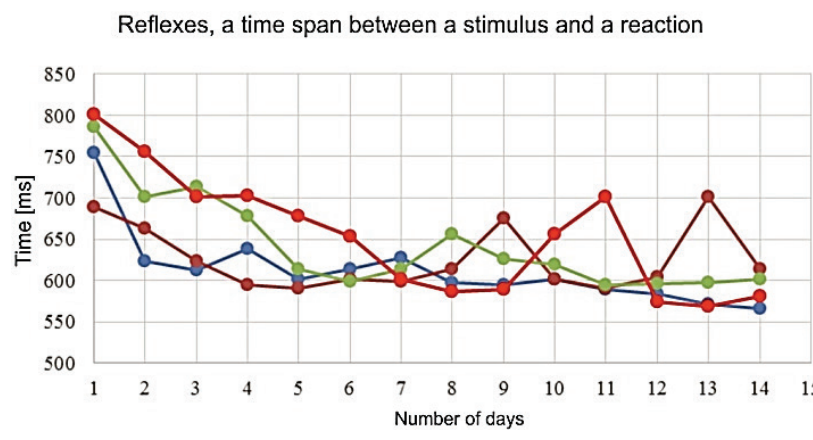

$\longrightarrow$ Person $1 \rightarrow$ Person $2 \multimap$ Person $3 \multimap$ Person 4

Figure 18 Results of the response time exercises

\section{Auditory memory}

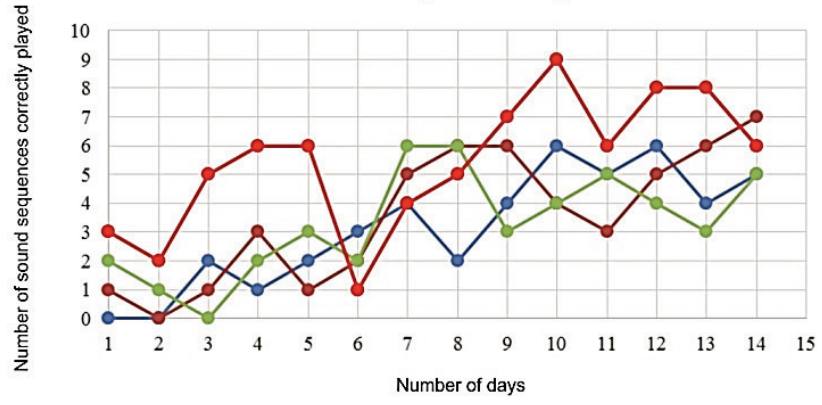

$\rightarrow$ Person $1 \rightarrow$ Person $2 \rightarrow$ Person $3 \rightarrow$ Person 4

Figure 19 Results of the auditory memory exercises

The last test was to verify the auditory memory exercise (Fig. 19). The vertical axis shows the number of correctly reproduced sound sequences in a row. The trend for all the persons was growing, which indicated that the exercises had a positive effect on their auditory memory.

\section{CONCLUSIONS}

The article presents an innovative device which allows to carry out diagnostics and rehabilitation of people who, as a result of their illnesses, have lost a full range and precision of the movements in their upper limbs, who experience problems with concentration, memory problems (visual and auditory) or poorer reflexes (the time period of correct response to a stimulus), especially those with a light degree of hemiparesis.

The original design of the device consists of a wooden base, and four wooden frames with sensors which detect interruptions to beams of infrared (IR) light. The user performs his tasks by inserting his hand in an appropriate frame and thus breaking an appropriate IR beam. When the IR sensor detects a hand in its field of operation, the user receives a feedback though a LED diode, telling him/her about the IR light beam being broken, which means that an element of biofeedback is also introduced into the rehabilitation process (i.e. stimulation with visual stimuli).

Another novelty of the system with a view to enhance the rehabilitation process is the distance sensor. It is 
mounted between the two middle frames and informs the user and the physiotherapist about the distance from the user to the device. It is very important to monitor the distance between the user and the device since a user who sits close to the unit engages different muscles (which are not necessarily those, which at this point should be diligently rehabilitated) than if he keeps a bigger distance.

Also, it is worth noting that the rehabilitation exercises in virtual reality are shown on the display screen and are additionally supported in reality with the four frames and also with the connectors, whose points of installation can be adjusted and thus create real obstacles, depending on the current condition of the patient.

Monitoring of the proper positioning of the upper limbs during the exercises and breaking the IR beams is made possible through a vision system, which also enables automatic recognition of a person and logging them in without having to manually select the user from a predefined database.

The device has been equipped with an original set of exercises, which help improve physical movements, memory (visual and auditory) and reflexes (reaction time to a stimulus).

The set of games for kids include a quiz, exercise on reflexes, on visual memory, on auditory memory, and a game involving playing music, which in particular has been dedicated to the youngest users.

Every exercise has been designed with a particular focus on providing biofeedback. The games have been developed while keeping in mind a need to train a given limb in a predefined space, and with the use of an additional stimulation such as visual information on the screen or via the LED diodes; or auditory stimulation through introducing music or the possibility to even make one's own interesting music through breaking the IR beams. It all enables recovering one's fitness at a faster pace.

An important element of the system is the reporting tab which enables us to regularly control the rehabilitation process, to introduce corrections to be applied both as to the choice of specific games to train specific skills, or as to make necessary adjustments to the program content or the time limit provided to complete a task. What is important, the device has been equipped with an intuitive user interface.

Another important advantage of the device is its versatility, i.e. the ability to be easily adjusted to cater for some other needs, for example to rehabilitate people with different diseases, or to make an educational stand for children, etc.

The verification and tests of the device took place in a kindergarten with children aged $5-7$ years. The device proved very popular both among children and among their teachers. The control system, which uses sensors and enables breaking IR beams seemed very attractive and mobilized children to want to participate in exercises.

It should also be highlighted that the device can be easily expanded and adapted to match the expectations of individual customers. For example, extra windows and sensors can be fitted, which will enrich the therapy program. Also, it is worth noting that the device is produced entirely of wood. Thanks to its size and weight, the device is portable and can be easily transported to patients' homes, which greatly facilitates work of physiotherapists and expands the range of their services outside health centers.

The innovative features and the unconventional way of running exercises with the presented device is further proven by the fact that a patent application No P.419184 and P.419183 for this device to rehabilitate one's physical and learning abilities has been filed.

\section{Acknowledgements}

This work was supported in part by the Vice-Rector for Research the Rzeszow University of Technology (U778/DS/M).

\section{REFERENCES}

[1] Hayward, K., Barker, R., \& Brauer, S. (2010). Interventions to promote upper limb recovery in stroke survivors with severe paresis: a systematic review. Disability and Rehabilitation, 32(24), pp. 1973-1986. https://doi.org/10.3109/09638288.2010.481027

[2] Levine, P. G. (2008). Stronger after stroke: your roadmap to recovery. Read How You Want, New York.

[3] Stroke Association, Research Spend in the UK. (2014). Comparing stroke, cancer, coronary heart disease and dementia. http://www.stroke.org.uk/research-spend-uk. (Accessed on 22.09.2016)

[4] Gijbels, D., Lamers, I., Kerkhofs, L., Alders, G., Knippenberg, E., \& Feys, P. (2011). The ArmeoSpring as training tool to improve upper limb functionality in multiple sclerosis: a pilot study. Journal of Neuro Engineering and Rehabilitation, 8(5), 2-8. https://doi.org/10.1186/1743-0003-8-5

[5] He, J., Balasubramanian, S., \& Wei, R. (2008). Designing interactive and intelligent control for rehabilitation robots. ICIRA 2008 Springer-Verlag, Berlin, 7-16. https://doi.org/10.1007/978-3-540-88518-4_2

[6] Hesse, S., Schmidt, H., \& Werner, C. (2006). Machines to support motor rehabilitation after stroke: 10 years of experience in Berlin. Journal of Rehabilitation Research \& Development, 43(5), 671-678. https://doi.org/10.1682/JRRD.2005.02.0052

[7] Hesse, S., Schulte-Tigges, G., Konrad, M., Bardeleben, A., \& Werner, C. (2003). Robot - assisted arm trainer for the passive and active practice of bilateral forearm and wrist movements in hemiparetic subjects. Archives Physical Medicine and Rehabilitation, 84, 915-920. https://doi.org/10.1016/S0003-9993(02)04954-7

[8] Krebs, H. I., Hogan, N., Aisen, M. L., \& Volpe, B. T. (1998). Robot - aided neurorehabilitation. IEEE Transactions on Rehabilitation Engineering, 6, 75-87. https://doi.org/10.1109/86.662623

[9] Šekoranja, B., Jerbić, B., \& Šuligoj, F. (2015). Virtual surface for human-robot interaction. Transactions of FAMENA, 39(1), 53-64.

[10] Kos, N. (2016). Patients with brain trauma and brain diseases - the meaning of early rehabilitation. Fizikalna $i$ rehabilitacijska medicina, 28(1-2), 151-160.

[11] Giustini, A. (2011). Neurorehabilitation: management and outcomes in physical and rehabilitation network. Fizikalna $i$ rehabilitacijska medicina, 22(1-2), 1-10.

[12] Gunasekara, M., Gopura, R., \& Jayawardena, S. (2015). 6REXOS: upper limb exoskeleton robot with improved pHRI. International Journal of Advanced Robotic Systems, 4, 1-13. https://doi.org/10.5772/60440

[13] Maciejasz, P., Eschweiler, J., Gerlach-Hahn, K., JansenTroy, A., \& Leonhardt, S. (2014). A survey on robotic 
devices for upper limb rehabilitation. Journal of Neuro Engineering and Rehabilitation, 11(3), 1-29. https://doi.org/10.1186/1743-0003-11-3

[14] Lo, H. S. \& Xie, S. Q. (2012). Exoskeleton robots for upperlimb rehabilitation: State of the art and future prospects. Medical Engineering \& Physics, 34(3), 261-268. https://doi.org/10.1016/j.medengphy.2011.10.004

[15] Pistohl, T., Joshi, D., Ganesh, G., Jackson, A., \& Nazarpour, K. (2015). Artificial proprioceptive feedback for myoelectric control. IEEE Transactions on Neural Systems and Rehabilitation Engineering, 23(3), 498-507. https://doi.org/10.1109/TNSRE.2014.2355856

[16] Ozkul, F. \& Barkana, D. E. (2013). Upper-extremity rehabilitation robot RehabRoby: methodology, design, usability and validation. International Journal of Advanced Robotic Systems, 10(1), 1-13. https://doi.org/10.5772/57261

[17] Stein, J., Harvey, R. L., Macko, R. F., Winstein, C. J., \& Zorwitz, R. D. (2008). Stroke recovery andrehabilitation. Demos Medical.

[18] Ciszewski, M., Buratowski, T., Giergiel, M., Małka, P., \& Kurc, K. (2014). Virtual prototyping, design and analysis of an in-pipe inspection mobile robot. Journal of Theoretical and Applied Mechanics, 52(2), 417-429.

[19] Kurc, K., Szybicki, D., Burghardt, A., \& Muszyńska, M. (2016). The application of virtual prototyping methods to determine the dynamic parameters of mobile robot. Open Engineering, 6(1), 55-63. https://doi.org/10.1515/eng-2016-0006

[20] Kohut, P., Kurc, K., Szybicki, D., Cioch, W., \& Burdzik, R. (2015). Vision based motion analysis and deflection measurement of a robot's crawler unit. Journal of Vibroengineering, 17(8), 4112-4121.

[21] Tutak, J. S. (2016). Design of ELISE robot for the paretic upper limb of stroke survivors. Journal of Vibroengineering, 18(6), 4069-4085. https://doi.org/10.21595/jve.2016.16812

[22] Novak-Marcincin, J., Brazda, P., Janak, M., \& Kocisko, M. (2011). Application of virtual reality technology in simulation of automated workplaces. Tehnicki vjesnik Technical Gazette, 18(4), 577-580

[23] Fain, N., Kline, M., Vukasinovic, N., \& Duhovnik, J. (2010). The impact of management on creativity and knowledge transfer in an academic virtual enterprise. // Tehnicki vjesnik - Technical Gazette, 17(3), 347-351.

[24] Dong-Yuan, G., Xi-Fan, Y., Qing-He, Y., \& Hong, J. (2014). Robot sensor calibration via neural network and particle swarm optimization enhanced with crossover and mutation. Tehnicki vjesnik - Technical Gazette, 21(5), 1025-1033.

[25] Iqbal, J., Ullah, M. I., Khan, A. A., \& Irfan, M. (2015). Towards sophisticated control of robotic manipulators: an experimental study on a pseudo-industrial arm. Strojniški vestnik - Journal of Mechanical Engineering, 61(7-8), 465470. https://doi.org/10.5545/sv-jme.2015.2511

[26] Giergiel, J. \& Kurc, K. (2011). Identification of the mathematical model of an inspection mobile robot with fuzzy logic systems and neural networks. Journal of Theoretical and Applied Mechanics, 49(1), 209-225.

[27] Hendzel, Z. \& Szuster, M. (2011). Discrete neural dynamic programming in wheeled mobile robot control. Communications in Nonlinear Science and Numerical Simulation, 16(5), 2355-2362. https://doi.org/10.1016/j.cnsns.2010.04.046

[28] Reinkensmeyer, D., Pang, C., Nessler, J., \& Painter, C. (2001). Java therapy: web-based roboticrehabilitation. Integration of Assistive Technology Research Series (Assistive Technology Research Series), 9, 66-71.

\section{Contact information:}

Jacek S. TUTAK, PhD, Eng

Rzeszow University of Technology,

Dept. of Applied Mechanics and Robotics,

al. Powstańców Warszawy 8, 35-959 Rzeszow, Poland

tutak.j@prz.edu.p

Wojciech KOŁODZIEJ, MSc Eng.

Rzeszow University of Technology,

Dept. of Applied Mechanics and Robotics,

al. Powstańców Warszawy 8, 35-959 Rzeszow, Poland allegrowodec@wp.pl 